\title{
Catalytic Dehydration of Glycerol under Mild Condition: An Environmentally Benign Acrolein Production
}

\author{
Ágnes Zsigmond $^{1}$, Péter Bata ${ }^{1}$, Mónika Fekete ${ }^{1}$, Ferenc Notheisz ${ }^{2}$ \\ ${ }^{1}$ Department of Organic Chemistry, University of Szeged, Szeged, Hungary; ${ }^{2}$ Organic Catalysis Research Group of the Hungarian \\ Academy of Sciences, University of Szeged, Szeged, Hungary. \\ Email: \{azsig, notheisz\}@chem.u-szeged.hu, bata_peter@freemail.hu
}

Received February $8^{\text {th }}, 2010$; revised March 29 $9^{\text {th }}, 2010$; accepted March $31^{\text {st }}, 2010$.

\begin{abstract}
The increase of biodiesel production results in the accumulation of glycerol, which requires an increasing demand towards the study of chemical application of glycerol. Glycerol has to be transformed to other valuable chemicals, which can be used as starting materials for organic synthesis. With the final goal to find a reasonable solution for this problem we have studied the dehydration of glycerol in liquid phase using a supported HPA catalyst and developed an environmentally benign production of acrolein. Our method does not have any extreme conditions and produces a total conversion with high (93\%) selectivity.
\end{abstract}

\section{Keywords: Consuming of Excess Glycerol, Production of Acrolein, Heterogeneous Method}

\section{Introduction}

Since people around the world almost exclusively cover its increasing energy demand with fossil basic materials (crude oil, natural gas, coal), sooner or later we are going to run out of these materials. To avoid this situation, the only possibility is to use biomass as a carbon source for the synthesis of fuels [1]. However, the use of biomass industrially in economically relevant chemical synthesis, new processes have to develop so that crude oil can be substituted [2]. Crude oil can be substituted by biodiesel where rape-oil is converted with methanol.

However, there is a problem accompanying the biodiesel production, namely the increase of the production of glycerol as a by-product, which can cause environmental and economical problems, as well. The effective utilization of this byproduct will be a key issue to promote the bio-diesel commercialization. Consequently an increasing demand has been developed towards the study of chemical application of glycerol to other valuable chemicals, which can be used as starting materials for organic synthesis [3].

One of the possibilities is to perform an acid-induced dehydration of glycerol to acrolein, as it can be seen in Scheme 1.

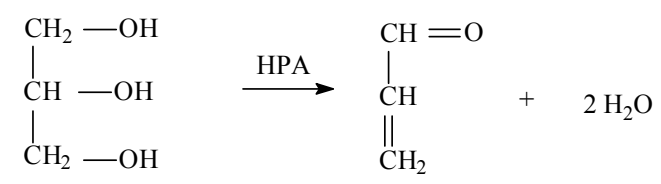

Scheme 1. Heteropolyacid catalyzed dehydration of glycerol to acrolein
Acrolein is an important chemical intermediate so it is very important to develop a sustainable and costeffective production route via glycerol from biodiesel, which offers an alternative for the presently dominating petrochemical process based on propylene.

The dehydration of glycerol to acrolein became a relatively well studied subject recently, when the biodiesel production started to grow. However, most of these works applying drastic conditions, e.g. the dehydration of glycerol in sub- and supercritical water. Bühler et al. found only low glycerol conversion (31\%) and similar selectivity (37 mol\%) meanwhile they used relatively high pressure $(25-45 \mathrm{MPa})$ and temperature $\left(300-400^{\circ} \mathrm{C}\right)$ [4].

Ramayya et al. have studied the effect of the addition of sulfuric acid on the dehydration of glycerol and found that the addition of acid dramatically enhances the acrolein yield [5]. They have also used high temperature $\left(300-350^{\circ} \mathrm{C}\right)$ and pressure $(34.5 \mathrm{MPa})$ and the obtained acrolein conversion was not too high. The other disadvantage of this method was the application of the highly corrosive sulfuric acid.

Meanwhile it was proven that high reaction temperature was necessary for the dehydration of glycerol, since no acrolein formation was detected at $250^{\circ} \mathrm{C}, 34.5 \mathrm{MPa}$ using sulfuric acid as a catalyst [6].

Some other work can be found dealing with gas phase dehydration of glycerol on mesoporous ZSM-5 [7], or $\mathrm{Nb}_{2} \mathrm{O}_{5}$ catalyst [8], but either the low selectivity or the expensive catalyst makes these methods practically un- 
applicable.

H. Atia, et al. have also studied the gas phase dehydration of glycerol to acrolein using different supported HPA, as catalysts $[9,10]$. The influence of selected support materials, catalyst loading and reaction temperature on acrolein formation was studied at standardized reaction conditions. Alumina was found to be superior to silica as support material with regard to catalyst activity and selectivity.

Some other works were dealing with also the gas phase dehydration of glycerol using zirconia and silica supported heteropoly acid catalysts [11-13]. The authors compared the different catalyst preparation methods and they found that independently of the preparation history of $\mathrm{ZrO}_{2}$, the Keggin-anion at the catalyst surface appeared as a key to the selectivity for acrolein production. Acrolein selectivity as high as $70 \mathrm{~mol} \%$ was obtained over the catalysts having the intermediate densities (0.18-0.65 HPA nm$\left.{ }^{-2}\right)$.

The dehydration of glycerol to produce acrolein was performed over several solid acids [14,15]. Supported heteropoly acids were effective catalysts for the dehydration of glycerol. The catalytic activity depended on the types of heteropoly acid and on the size of the mesopores in the silica support. Silicotungstic acid supported on silica with mesopores of $10 \mathrm{~nm}$ showed the highest catalytic activity with the acrolein selectivity of $>85 \mathrm{~mol} \%$ at an ambient pressure and $275^{\circ} \mathrm{C}$.

According to our knowledge there is no environmentally benign process for the utilization of the excess glycerol. With the final goal to develop an environmentally benign production of acrolein, we have prepared a supported HPA catalyst and studied the dehydration of glycerol in liquid phase. For our study relatively mild conditions were selected and a new heterogeneous catalyst, which could replace the very corrosive sulfuric acid in this process.

\section{Experimental}

\subsection{Catalyst Preparation}

$7.0 \mathrm{~g}$ of $\mathrm{Al}_{2} \mathrm{O}_{3}$ was suspended in $30 \mathrm{~mL}$ of methanol with stirring. $1.93 \mathrm{~g}(0.67 \mathrm{mmol})$ of phosphotungstic acid hydrate (PTA) was dissolved in $25 \mathrm{~mL}$ of methanol and this solution was added dropwise into the alumina suspension with efficient stirring. The stirring was continued for an additional hour at room temperature, under an Ar atmosphere. The solution was removed from the solid material and the solid residue was washed with methanol several times to remove the weakly adsorbed PTA from the surface of $\mathrm{Al}_{2} \mathrm{O}_{3}$.

The prepared catalyst was characterized by FT-IR spectroscopy, taking the spectrum of the phosphotungstic acid hydrate and the anchored $\mathrm{PTA} / \mathrm{Al}_{2} \mathrm{O}_{3}$, as well. The FT-IR spectra were recorded on a Bio-Rad FTS-65 A spectrophotometer, in the range of $400-4000 \mathrm{~cm}^{-1}$, in $\mathrm{KBr}$ pellets.

\subsection{Dehydration Experiments}

The reaction was carried out in a two neck round-bottom flask, equipped with a dropping funnel and a column. The dried catalyst $(8.93 \mathrm{~g})$ was suspended in $70 \mathrm{ml}$ high boiling point solvent (diesel oil) in a round-bottom flask and was heated gradually to $300^{\circ} \mathrm{C}$ with efficient stirring. To keep the high temperature, the flask was covered with glass wool. The column was connected to a distillation apparatus and the distilled product was collected in a flask, cooled by ice. Having reached the suitable temperature $10 \mathrm{~mL}(0.13 \mathrm{~mol})$ of glycerol was dropped slowly, meanwhile the product was distilled out. The two phase product was separated and the organic phase was dried by $\mathrm{MgSO}_{4}$. The water phase was extracted by ether and the product distribution of both phases was determined by HP 5890 GC, using HP1 column and ethanol inert standard.

\section{Results and Discussion}

\subsection{Catalyst Characterization}

With the final goal of developing an environmentally friendly process for the utilization of glycerol, which was produced in a large excess in the biodiesel production, we have anchored the phosphotungstic acid hydrate on $\mathrm{Al}_{2} \mathrm{O}_{3}$ support. The prepared catalyst was characterized by FT-IR spectroscopy, taking the spectrum of the free phosphotungstic acid hydrate and the anchored PTA, as well (Figure 1).

The comparison of the two spectra above shows convincingly that the "free" acid and the heterogenized samples have several similar bands (1090 and $\left.890 \mathrm{~cm}^{-1}\right)$, indicating the anchoring of the same acid.

\subsection{Dehydration Experiments}

The preliminary experiments were done using $\mathrm{H}_{3} \mathrm{PO}_{4} /$ $\mathrm{Al}_{2} \mathrm{O}_{3}$ catalyst, which was prepared according to a patent description [16]. For the preparation of acrolein the same protocol was used as described in the patent and our preliminary experiments showed an increase in acrolein selectivity with increasing temperature. The dehydration reaction has not started below $240^{\circ} \mathrm{C}$, and it seems that a good acrolein production needs at least $280^{\circ} \mathrm{C}$ or even higher temperature. In other words, the most difficult problem in the laboratory to find a good, neutral solvent with a high boiling point. We have tried several solvents e.g. triglykoldimethyl ether, methyldiglykol-tert-butyl ether and finally a distilled fraction of diesel oil was selected.

Based on data of our preliminary experiments and 


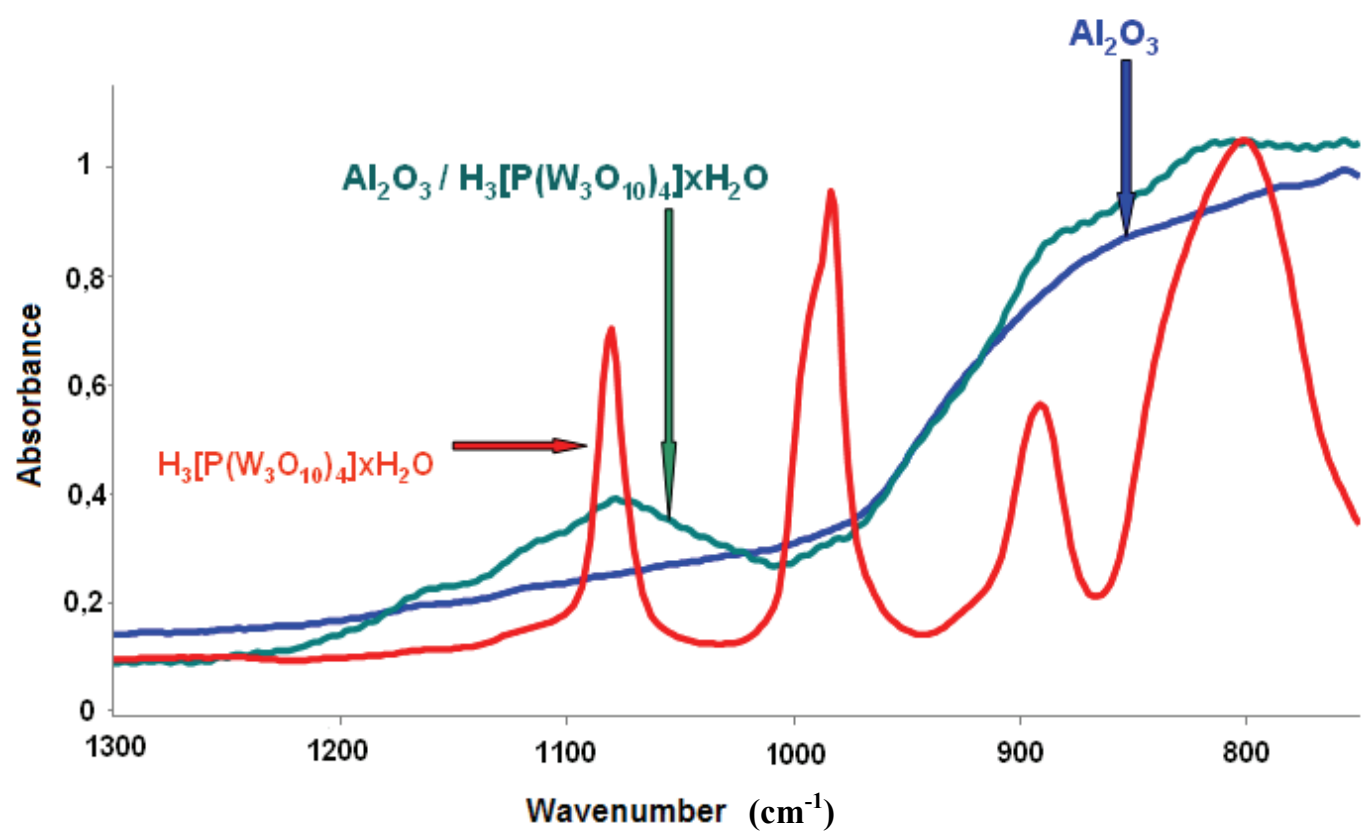

Figure 1. The FT-IR spectra of the "free" and anchored phosphotungstic acid hydrate

Table 1. Comparison of $\mathrm{H}_{3} \mathrm{PO}_{4} / \mathrm{Al}_{2} \mathrm{O}_{3}$ and $\mathrm{H}_{3}\left[\mathrm{P}\left(\mathrm{W}_{2} \mathrm{O}_{10}\right)_{4}\right] / \mathrm{Al}_{2} \mathrm{O}_{3}$ catalysts

\begin{tabular}{ccccc}
\hline Catalyst & Run & $\begin{array}{c}\text { Temperature } \\
\left(\mathbf{(}^{\circ} \mathbf{C}\right)\end{array}$ & $\begin{array}{c}\text { Conversion } \\
(\mathbf{\%})\end{array}$ & $\begin{array}{c}\text { Yield } \\
(\mathbf{\%})\end{array}$ \\
\hline $\mathrm{H}_{3} \mathrm{PO}_{4} / \mathrm{Al}_{2} \mathrm{O}_{3}$ & 1 & 260 & 9.7 & 17.6 \\
$\mathrm{H}_{3} \mathrm{PO}_{4} / \mathrm{Al}_{2} \mathrm{O}_{3}$ & 2 & 260 & 10.1 & 18.3 \\
$\mathrm{H}_{3}\left[\mathrm{P}_{2}\left(\mathrm{~W}_{2} \mathrm{O}_{10}\right)_{4}\right] / \mathrm{Al}_{2} \mathrm{O}_{3}$ & 3 & 260 & 31.2 & 25.5 \\
$\mathrm{H}_{3}\left[\mathrm{P}\left(\mathrm{W}_{2} \mathrm{O}_{10}\right)_{4}\right] / \mathrm{Al}_{2} \mathrm{O}_{3}$ & 4 & 260 & 30.9 & 25.4 \\
\hline
\end{tabular}

former experience, we have selected an other catalyst, which was the phosphotungstic acid hydrate. Using the former protocol with a higher boiling point of solvent and a more active catalyst we have expected a higher conversion, as well. The comparison of the two catalysts can be seen in Table 1.

Our expectation was correct as it can be seen in Table 1, with the new catalyst and a higher boiling point of solvent we obtained about three times higher conversion and selectivity was also higher. Consequently, studying the effect of temperature on the dehydration reaction we have decided to use these conditions (catalyst, solvent).

In the third set of the experiments we have studied the effect of temperature on the reaction, more precisely we wanted to determine the optimum temperature for the formation of acrolein. Having the same reaction conditions we have changed systematically the temperature and the results can be seen in Table 2.

The systematic increase in temperature has increased significantly the yield of acrolein, at $300^{\circ} \mathrm{C}$ we have reached $97 \%$ conversion and also a high $77.5 \%$ selectivity. Another increase in temperature with $20^{\circ} \mathrm{C}$ could produce the total conversion and an even higher $92.8 \%$ selectivity.

Applying a good acidic catalyst and a high boiling point solvent we could determine the optimum conditions for the dehydration of glycerol. Using the above conditions we could produce total conversion and very high (93\%) selectivity, which are the best values-according to our knowledge - so far in the literature. Meanwhile we could not observe any other dehydration products (e.g. acetic acid, acetaldehyde).

Table 2. The dehydration of glycerol on $\mathrm{H}_{3}\left[\mathrm{P}\left(\mathrm{W}_{2} \mathrm{O}_{10}\right)_{4}\right] / \mathrm{Al}_{2} \mathrm{O}_{3}$ catalysts

\begin{tabular}{cccc}
\hline Run & $\begin{array}{c}\text { Temperature } \\
\left({ }^{\mathbf{0}} \mathbf{C}\right)\end{array}$ & $\begin{array}{c}\text { Conversion } \\
(\mathbf{\%})\end{array}$ & $\begin{array}{c}\text { Yield } \\
(\mathbf{\%})\end{array}$ \\
\hline 5 & 260 & 31.2 & 25.5 \\
6 & 260 & 30.9 & 25.4 \\
7 & 280 & 52.1 & 56.4 \\
8 & 280 & 53.9 & 56.6 \\
9 & 300 & 97.0 & 77.5 \\
10 & 300 & 98.0 & 78.0 \\
11 & 320 & 100 & 92.8 \\
12 & 320 & 100 & 92.5 \\
\hline
\end{tabular}


The other advantage of this method that it is not applying drastic conditions- high pressure and temperatureas the methods described in the literature. So, using the above conditions we could develop an environmentally benign method for the utilization of the extra amount of glycerol produced by the biodiesel formation.

To check the efficiency of our catalyst we have made a blind experiment, too. During the blind experiment we have followed the same protocol without catalyst and we have not observed any acrolein formation. In other words applying these conditions the thermal dehydration is not occurring.

\subsection{Recycling of the $\mathrm{H}_{3}\left[\mathrm{P}\left(\mathrm{W}_{2} \mathrm{O}_{10}\right)_{4}\right] / \mathrm{Al}_{2} \mathrm{O}_{3}$ Catalyst}

It is well known that the most important advantage of heterogeneous catalysts is their recyclability. To check the reusability of our catalyst, we have applied our catalyst in three subsequent runs and the results can be seen on Figure 2.

As it can be seen on Figure 2, (light column represents conversion, dark column selectivity) we could apply successfully our catalyst in three subsequent runs. Neither the conversion nor the selectivity has changed during the three subsequent experiments, which means that our catalyst has the advantage of the heterogeneous catalyst.

The material balance is a very important fact from the point of view the practical application. For the experiments we have used $10 \mathrm{ml}(0.13 \mathrm{~mol})$ of glycerol for each run and the amount of isolated acrolein has increased as a function of temperature.

Table 3 clearly shows that the isolated amount of acrolein has increased with increasing temperature. At the optimum temperature the material balance is reasonable. The loss of material is about $10 \%$, which means the method is still practically useful.

\section{Conclusions}

With the final goal of the utilization of the extra amount of glycerol, produced by the biodiesel production, we

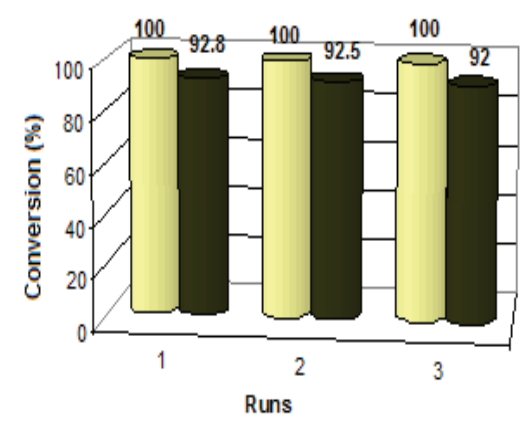

Figure 2. The selectivity and conversion in three subsequent runs at $300^{\circ} \mathrm{C}$
Table 3. The material balance as a function of temperature

\begin{tabular}{cccc}
\hline $\begin{array}{c}\text { Temparature } \\
\left({ }^{\circ} \mathbf{C}\right)\end{array}$ & \multicolumn{2}{c}{$\begin{array}{c}\text { Amount of acrolein } \\
(\mathbf{g})\end{array}$} & $\begin{array}{c}\text { Yield } \\
(\mathbf{m o l})\end{array}$ \\
\hline $\mathbf{2 6 0}$ & 0.84 & 0.02 & 15.38 \\
$\mathbf{2 8 0}$ & 4.04 & 0.07 & 53.84 \\
$\mathbf{3 0 0}$ & 5.80 & 0.10 & 76.92 \\
$\mathbf{3 2 0}$ & 6.69 & 0.12 & 92.30 \\
\hline
\end{tabular}

have developed a method to prepare valuable starting material. This starting material is the acrolein, having two reactive double bonds, which make it possible to apply in various organic synthesis. The method developed by us is an environmentally benign method, does not have any extreme conditions and producing a total conversion with high (93\%) selectivity. According to our knowledge this is the only environmentally benign method which produces such a high conversion and selectivity.

\section{REFERENCES}

[1] P. Gallezot, "Process Options for Converting Renewable Feedstocks to Bioproducts," Green Chemistry, Vol. 9, No. 4, 2007, pp. 295-302.

[2] A. Corma, S. Iborra and A. Velty, "Chemical Routes for the Transformation of Biomass into Chemicals," Chemical Reviews, Vol. 107, No. 6, 2007, pp. 2411-2502.

[3] C.-H. Zhou, J. N. Beltramini, Y.-X. Fan and G. Q. Lu, "Chemoselective Catalytic Conversion of Glycerol as a Biorenewable Source to Valuable Commodity Chemicals," Chemical Society Reviews, Vol. 37, No. 3, 2008, pp. 527549.

[4] W. Bühler, E. Dinjus, H. J. Ederer, A. Kruse and C. Mas, "Ionic Reactions and Pyrolysis of Glycerol as Competing Reaction Pathways in Near- and Supercritical Water," Journal of Supercritical Fluids, Vol. 22, No. 1, 2002, pp. 37-53.

[5] S. Ramayya, A. Brittain, C. DeAlmeida, W. Mok and M. J. Antal, "Acid-Catalysed Dehydration of Alcohols in Supercritical Water," Fuel, Vol. 66, No. 10, 1987, pp. 1364-1371.

[6] M. Watanabe, T. Iida, Y. Aizawa, T. M. Aida and H. Inomata, "Acrolein Synthesis from Glycerol in Hot-Compressed Water," Bioresource Technology, Vol. 98, No. 6, 2007, pp. 1285-1290.

[7] C. Márquez-Alvarez, E. Sastre and J. Pérez-Pariente, "Solid Catalysts for the Synthesis of Fatty Esters of Glycerol, Polyglycerols and Sorbitol from Renewable Resources Sorbitol from Renewable Resources," Topics in Catalysis, Vol. 27, No. 1-4, 2004, pp. 105-117.

[8] S.-H. Chai, H.-P. Wang, Y. Liang and B.-Q. Xu, "Sustainable Production of Acrolein: Gas-Phase Dehydration of Glycerol over $\mathrm{Nb}_{2} \mathrm{O}_{5}$ Catalyst," Journal of Catalysis, Vol. 250, No. 2, 2007, pp. 342-349.

[9] H. Atia, U. Armbruster and A. Martin, "Dehydration of Glycerol in Gas Phase Using Heteropolyacid Catalysts as Active Compounds," Proceedings of the DGMK-Confe- 
rence "Future Feedstocks for Fuels and Chemicals", Berlin, 29 September-1 October 2008, pp. 177-184.

[10] H. Atia, U. Armbruster and A. Martin, "Dehydration of Glycerol in Gas Phase Using Heteropolyacid Catalysts as Active Compounds," Journal of Catalysis, Vol. 258, No. 1, 2008, pp. 71-82.

[11] S.-H. Chai, H.-P. Wang, Y. Liang and B.-Q. Xu, "Sustainable Production of Acrolein: Preparation and Characterization of Zirconia-Supported 12-Tungstophosphoric Acid Catalyst for Gas-Phase Dehydration of Glycerol," Applied Catalysis A: General, Vol. 353, No. 2, 2009, pp. 213-222.

[12] S.-H. Chai, H.-P. Wang, Y. Liang and B.-Q. Xu, "Sustainable Production of Acrolein: Investigation of Solid Acid-Base Catalysts for Gas-Phase Dehydration of Glycerol," Green Chemistry, Vol. 9, No. 10, 2007, pp. 11301136.
[13] S.-H. Chai, H.-P. Wang, Y. Liang and B.-Q. Xu, "Sustainable Production of Acrolein: Gas-Phase Dehydration of Glycerol over 12-Tungstophosphoric Acid Supported on $\mathrm{ZrO}_{2}$ and $\mathrm{SiO}_{2}$," Green Chemistry, Vol. 10, No. 10, 2008, pp. 1087-1093.

[14] L. Ning, Y. Ding, W. Chen, L. Gong, R. Lin, L. Yuan and Q. Xin, "Glycerol Dehydration to Acrolein over Activated Carbon-Supported Silicotungstic Acids," Chinese Journal of Catalysis, Vol. 29, No. 3, 2008, pp. 212-214.

[15] E. Tsukuda, S. Sato, R. Takahashi and T. Sodesawa, "Production of Acrolein from Glycerol over Silica-Supported Heteropoly Acids," Catalysis Communications, Vol. 8, No. 9, 2007, pp. 1349-1353.

[16] H. E. Hoyt and T. H. Manninen, "Production of Acrolein from Glycerol," U.S. Patent 2558520, U.S. Industrial Chemicals, Arvada, 1948. 